\title{
"Simbiter Omega" Multiprobiotic: A New Aid in Treatment of Generalized Chronic Periodontitis
}

\author{
Mykytenko AO ${ }^{1}$, Yankovskiy $\mathrm{DS}^{2}$, Dyment GS ${ }^{2}$, Beregova $\mathrm{TV}^{3}$ and Neporada $\mathrm{KS}^{1 *}$ \\ ${ }^{1}$ Ukrainian Medical Dental Academy, Poltava, Ukraine \\ 2"O.D. Prolisok" scientific production company, Kyiv, Ukraine \\ ${ }^{3}$ The Institute of Biology, Taras Shevchenko National University of Kyiv, Kyiv, Ukraine
}

\begin{abstract}
Received: December 07, 2014; Accepted: March 25, 2015; Published: April 10, 2015
*Corresponding author: Neporada KS, Professor, Department of Medicinal, Bioorganic and Biological Chemistry, Ukrainian Medical Dental Academy, 36011, 23 Shevchenko Street, Poltava, Ukraine, Tel: +380-956-015-918; E-mail: neporada_69@mail.ru
\end{abstract}

\begin{abstract}
Contemporary treatment of generalized chronic periodontitis is based on elimination of periodontal pathogens by antibiotics and antiseptics after local scaling and administration of cytoprotectors, immunomodulators and osteotrophic drugs for oral rehabilitation. Current concept distinguishes three levels of protection: the biofilm, which normally should be in dynamic equilibrium of competitive antagonism of periodontal pathogens and symbiotic normoflora; a natural barrier, presented by oral mucosa epithelium; the system of general and tissue immunity. Under certain circumstances, degradation of one of the protective layer leads to imbalance and total insufficient protection. Consequently, we hypothesize that such therapy will result in destruction of the first protective layer, i.e., oral microbiota microbiocoenosis imbalance. Therefore, monotherapy with "Simbiter omega" multiprobiotic is the effective supplement for common therapy, since it promotes restoration of all oral mucosa protective layers against the background of antagonist fighting of colonies of Bifidobacterium, Lactobacillus, Lactococcus, Streptococcus, Propionibacterium and Acetobacter with periodontal pathogens Actinobacillusactynomycetemcomitans, Porphyromonasgingivalis, Bacteroidesforsythus mainly. Omega-3 and omega- 6 essential polyene fatty acids and smectite, containing in multiprobiotic, have synergizing parodontoprotective effect.
\end{abstract}

Keywords: Multiprobiotic; Probiotic; Periodontitis; Smectite; Omega-3 essential polyene fatty acid

\section{Introduction}

The new era of periodontal microbiology began when Socransky S.S. and Haffajee A.D. (1992) proposed the new "formula" for periodontitis lesions. The essence of the concept was that periodontal disease is the complicated pathophysiological process, involving several factors: bacterial pathogens, sensible organism, imbalances of normal oral microflora and periodontal host response [1-3]. Consequently, oral dysbiosis is recognized as one of the main pathogenetic factors, leading to periodontitis. We hypothesize that treatment of inflammatory periodontal diseases may be effective in dysbiosis correction. Currently, the problem of microecologic disturbances is becoming global and, subsequently, there is a need in highly effective agents of antidysbiosis therapy. The demand for such products promoted avalanche-like enlargement of assortment, but, unfortunately, not all biocenose-regenerative agents are effective. The effective agent in treatment of periodontitis should posses the following properties. Firstly, the microorganism cultures are to be living and not lyophilized, in sufficient amount, as once they were entered to oral cavity, there is no time for activation, since the factors of periodontal pathogens aggression will immediately start eliminating them. Secondly, only mutual symbiosis with saccharolytic microorganisms can promote manifestation of rather efficient therapeutic effect as different levels of acidity, oxidation-reduction potential, content of oxygen, carbonic acids and nutrients are sustained in different regions of the oral cavity. Consequently, different regions of the oral cavity are populated with specific microbial associations for which the existent conditions are the most preferable [2]. Thirdly, microorganisms are to be competitive with periodontal pathogens as for receptors for adhesines at the surface of the microorganism cells. Fourthly, the agent should contain an effective enterosorbent in case of oral microbial ecology disturbances, as microbiocoenosis disturbance is normally accompanied by contamination of the organism by toxic compounds of both exogenous and endogenous nature. This is mostly caused by the loss of normoflora's fully protective function, which promotes neutralization and elimination of dangerous substances from the body, which penetrate from the ambient environment or are synthesized in the body due to metabolic processes disorder. Sanitation of biotope from allergens, mediators, and products of allergic or inflammatory reaction, metabolites, toxins, active peroxidates, viruses and other compounds promotes favorable conditions for vital functions of microbiota. Fifthly, to regenerate the oral cavity biofilm it is necessary to select the most suitable prebiotic for normobiome metabolism. Sixthly, it should be of cytoprotective action with respect to host cells. Probiotics of VII generation meets all these criteria. "Simbiter omega" multiprobiotic is one of the representatives of such agents. The study was aimed at clinical and biomedical validation of the effectiveness of "Simbiter omega" multiprobiotic administration to become a 
supplementary aid for the treatments of generalized chronic periodontitis.

\section{Methods}

The clinical and periodontal parameters of subjects were assessed by a single examiner. The clinical diagnosis of generalized chronic periodontitis was based on the presence of up to $30 \%$ of measured sites with Clinical Attachment Loss (CAL) > $5 \mathrm{~mm}$ [4]. 56 patients (mean age 35-42 years) have been involved into clinical study. The patients have been divided into following groups: 20 apparently healthy individuals were assigned into group I; patients with generalized chronic periodontitis of I-II degree in the remission period were assigned into group II. Patients from the main (II) group have undergone professional oral hygiene with subsequent prescription to use "Simbiter omega" multiprobiotic in dentoalveolar trays that resemble mouth guards overnight for 20 days. Dentoalveolar trays have reservoirs at marginal gingiva for direct contact of agent's symbiotic microflora with oral biofilm. They were made of polyethylene by the "Ultraform" dental vacuum composer (Figure 1).

"Simbiter omega" multiprobiotic has been developed by the "O.D. Prolisok" scientific production company, and is the new form of "Simbiter" group multiprobiotic of the VII generation. It comprises of 18 strains of probiotic bacteria that belong to Bifidobacterium, Lactobacillus, Lactococcus, Streptococcus, Propionibacterium groups and Acetobacter, and are in the form of steady mutual symbiosis. The base of the multiprobiotic is a high-purity smectite (bentonite) gel, which is a natural clay polymineral composition, $60-70 \%$ consisting of minerals from the montmorillonite group. Adsorbing and ion-exchange properties of smectites are considered to be the main factors of their therapeutic effect. The multiprobiotic has a positive effect on vital functions of saccharolytic obligate anaerobes, constituting its bacterial base, and human physiological microbiocoenosis. It also promotes the increase of mocroflora's resistance to difficult environments [5]. Additionally, the multiprobiotic consists of flax and wheat sprouts oils, which are the valuable source of Omega-3 and Omega- 6 essential polyene fatty acids. They reveal their cytoprotective and regulatory properties in the human body by restoration of the cytoderm and synthesis of mediators. Probiotic activity of the agent is determined by the high antagonistic activity relative to wide range of pathogenic and opportunistic microorganisms, synthesis of vitamins, short-chain fatty acids, exopolysaccharides, glycopeptides, etc. The "Simbiter omega" multiprobiotic contains not less than $2 \times 10^{10}$ live cells of probiotic bacteria in one doze $\left(10 \mathrm{~cm}^{3}\right)$ and is indicated to children above 3 years old and adults. One doze $\left(10 \mathrm{~cm}^{3}\right)$ of "Simbiter omega" contains concentrated biomass of microorganisms' symbiosis live cells: lactobacilli and lactococci not (less $1.0 \times 10^{10} \mathrm{CFU} / \mathrm{cm}^{3}$ ), propionic-acid bacteria (not less $1.0 \times 10^{9} \mathrm{CFU} / \mathrm{cm}^{3}$ ), bifidus bacteria (not less1.0 X $10^{10} \mathrm{CFU} / \mathrm{cm}^{3}$ ), vinegar bacteria (not less $1.0 \times 10^{6} \mathrm{CFU} / \mathrm{cm}^{3}$ ).

To estimate objectively the condition of periodontal tissues in patients of all groups the Fedorov-Volodkina's (1971) hygienic index (HI), Parma (1960) modification Papillary-MarginalAlveolar (PMA) index, gingival index (Schiller-Pisarev's test), Russel's (1956) Periodontal Index (PI), Mьhlemann and Mazor (1985) Sulcus Bleeding Index (SBI) have been calculated; Ketschke's test has been made to detect pus in periodontal recesses and the depth of periodontal recesses has been measured by the graduated probe; the degree of pathological tooth mobility has been determined [6].

Unstimulated oral fluid, collected from the patients on an empty stomach has been biochemically examined to measure the volume of oxidation-modified proteins [7], TBA-reactants [8], catalase [9] and superoxide dismutase [10] activity. The degree of endogenous intoxication has been determined by the volume of intermediate mass molecules [11]; proteinase-inhibitory potential has been measured through proteinase activity [12] and total antitryptic activity [13], ornithine decarboxylase activity [14].

Microbiological analysis of substance, withdrawn from periodontal recess was carried out by sterile pin \# 30 . Hereafter it was placed into hermetic container with the environment for preserving anaerobic microflora and delivered to the laboratory. Microbiological studies of periodontal recess's volume have
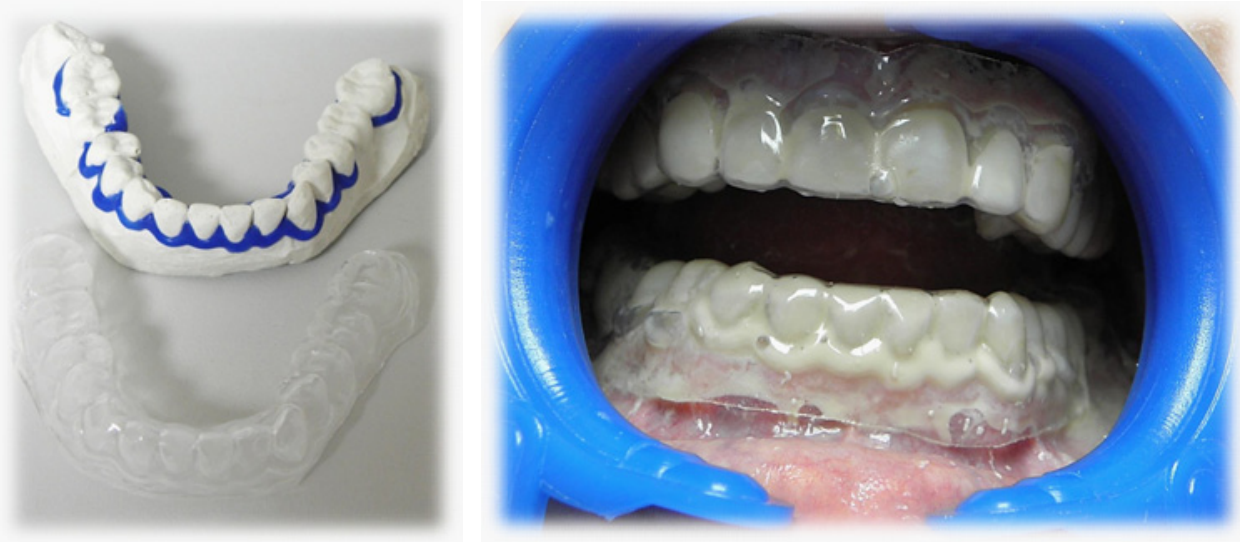

Figure 1: Study cast for manufacturing custom-made dentoalveolar tray with reservoir at the marginal gingiva to form a deposit zone. 

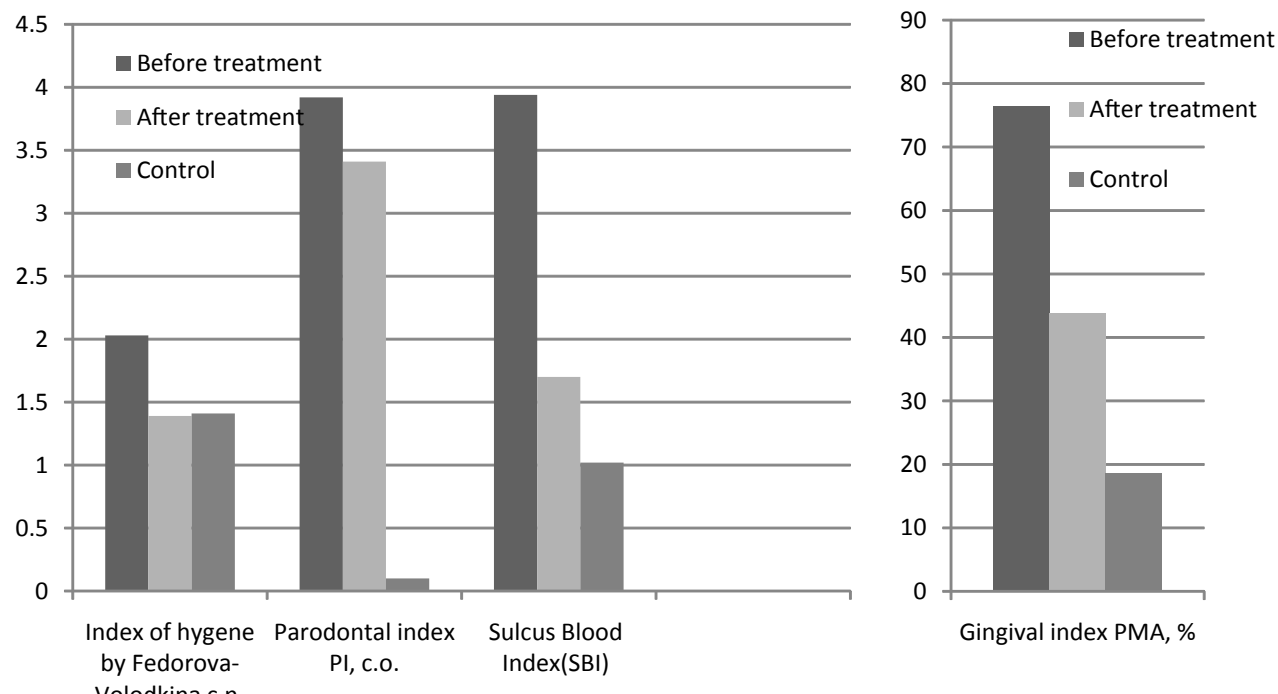

Figure 2: Index Estimation of Parodontal Status in Patients with Generalized Chronic Periodontitis of I-II Degree During the Remission Before and After Preventive Treatment with "Simbiter Omega" Multiprobiotic as Compared to Control Group, (M $\pm m$ ). Note: all data is statistically significant, $\mathrm{P}<0.05$.

been performed at Kharkiv I. I. Mechnikov Research Institute of Microbiology and Immunology of Ministry of Public Health of Ukraine in compliance with methodological guidelines [15].

During the test for distributional adequacy the Shapiro-Wilk's calculation criterion was applied. If the data corresponded to the normal distribution, then the authenticities of their differences, while comparing the mean values, were determined using the Student's t-criterion. In the case when data series were not subject to normal distribution, the statistical processing has been carried out using the Mann-Whitney's distribution-free method.

\section{Results}

The significant improvement of clinical indices used to estimate parodontal status in patients with generalized chronic periodontitis has been noted after prophylactic treatment with "Simbiter omega" multiprobiotic, as shown in Figure 2. The Fedorov-Volodkina's hygienic index, PMA gingival index, periodontal index and Sulcus Bleeding Index (SBI) were significantly changed in all patients after treatment as compared to the rates before "Simbiter omega" multiprobiotic administration (Figure 2).

Consequently, use of "Simbiter omega" multiprobiotic by all patients with generalized chronic periodontitis leads to inhibition of oxidative stress along with the increase of oral fluid antiradical protection.

Intermediate mass molecules reflect the degree of endogenous intoxication [11]. It has been revealed that the volume of intermediate mass molecules was significantly increased in oral fluid of patients with generalized chronic periodontitis. The volume of intermediate mass molecules has been significantly reduced in oral fluid of patients, administered with "Simbiter omega" multiprobiotic, as compared to the values before treatment (Figure 3).

Findings of microbiological examination, shown in Figure 3 , stated a significant reduction of periodontal pathogens after treatment, indicating about the clinical effectiveness of "Simbiter omega" multiprobiotic.

In conclusion, overnight administration of "Simbiter omega" multiprobiotic, applied under individual dentoalveolar caps, is the effective adjunctive therapy for patients with generalized chronic periodontitis, proved by the proteinase-inhibitory potential normalization, increased activity of ornithinedecarboxylase and inhibition of free radical oxidation along with antiradical protection of oral fluid.

\section{Discussion}

The objective estimation of proteolysis system is only possible in conditions of consideration of total proteolytic activity of oral fluid and proteinase inhibitors activity, which inhibits proteolytic enzymes and its ratio, is determined as proteinase-inhibitory potential.

Matrix Metallo Proteinases (MMPs) produced by immune regulatory cells and fibroblasts play a destructive role in the inflammatory periodontal lesion progression. It has been proven that HtrA1 plays a novel role in process of periodontal damage as proteolytic protein [3].

It has been established that total proteolytic activity significantly increased in oral fluid of all patients before treatment of generalized chronic periodontitis, whereas total antitryptic activity was decreasing. Therefore, the progression of generalized chronic periodontitis causes imbalance of oral fluid proteinase-inhibitory potential. Administration of multiprobiotic 


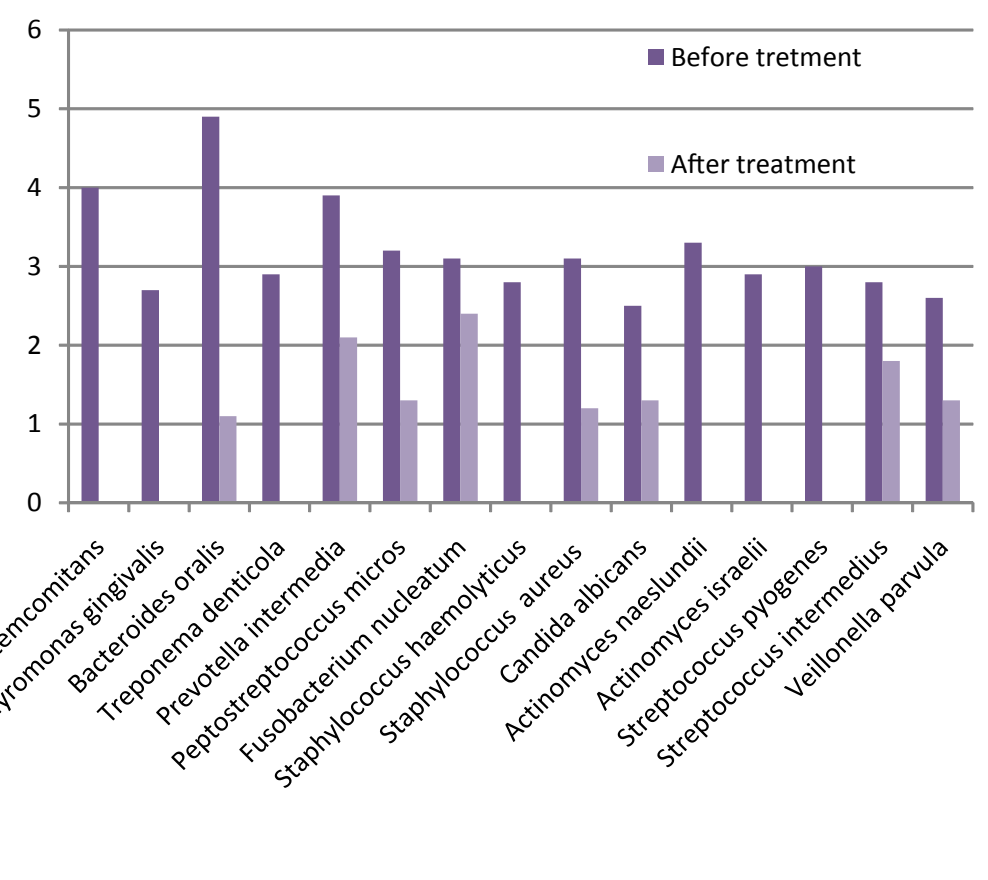

Figure 3: Biochemical Rates of Oral Fluid of Patients with Generalized Chronic Periodontitis of I-II Degree During the Remission Before and After Preventive Treatment with "Simbiter Omega" Multiprobiotic as Compared to Control Group, (M $\pm m)$.

Note: all data is statistically significant, $\mathrm{P}<0.05$.

promoted significant decrease of total proteolytic activity in oral fluid along with significant increase of total antitryptic activity in patients after treatment as compared to the values before treatment (Table 1).

Ornithinedecarboxylase is the key enzyme in synthesis of such regulatory polyamines as putrescine, spermine, spermidine, etc., which regulate the replication and transcription processes and, consequently, proliferation of cells. There is also evidence of the role of polyamines, related to ornithinedecarboxylase, in the Vascular Endothelial Growth Factor (VEGF) action mechanism $[16,17]$. In vitro studies show that polyamines stimulate the activity of DNA-dependent RNA- polymerase. Polyamines significantly initiate synthesis of peptides by changing the conformation of ribosomes. Consequently, polyamines play an important regulatory role in the processes related to biosynthesis of proteins and nucleic acids.

It has been established that ornithinedecarboxylase activity significantly decreased in oral fluid of all patients before treatment of generalized chronic periodontitis, as compared to control. After treatment ornithinedecarboxylase activity significantly increased in oral fluid of patients, who were administered with "Simbiter omega" multiprobiotic in dentoalveolar trays overnight, as compared to the activity of the enzyme before treatment (Table 1).

In sum, clinical efficacy of "Simbiter omega" multiprobiotic has been proved on the ground of proteinase-inhibitory potential investigation and ornithinedecarboxylase activity in oral fluid of patients with generalized chronic periodontitis with evidence of significant decrease of proteinase activity along with increase of activity of proteinase inhibitors and ornithinedecarboxylase. Admittedly, activation of processes of free radical oxidation is the universal mechanism of cell damage in stress situations $[18,19]$, ischemia and during post-ischemic period [20]. Lipid peroxidation products are recognized as one of the strongest modificators of biological membranes in the range of pathological processes. They are able to increase the penetrability of lipid biolayer for $\mathrm{K}^{+}, \mathrm{H}^{+}$and $\mathrm{Ca}^{2+}$ ions, change its physicochemical properties, leading to reduction of membrane fluidity, charge rate, causing the lipid-protein interaction disorder, inactivation of integral proteins [20].

The findings of integral indices of free radical oxidation of volume of oxidation-modified proteins and TBA-reactants in oral fluid during progression of generalized chronic periodontitis and treatment with "Simbiter omega" multiprobiotic are shown in Figure 3.

Intensification of free radical oxidation in oral fluid has been noted in all patients with generalized chronic periodontitis, which is indicated by significant increase of volume of oxidationmodified proteins and TBA-reactants. Administration of "Simbiter omega" multiprobiotic promotes significant decrease of volume of oxidation-modified proteins and TBA-reactants in oral fluid as compared to the values before treatment (Figure 3).

Catalase is one of the main enzymes of antiradical protection, able to inactivate peroxide, and is the synergist of superoxide dismutase; hence, estimation of its activities is important in 
Table 1: Biochemical values of oral fluid of patients with generalized chronic periodontitis of I-II degree during the remission before and after preventive treatment with "Simbiter Omega" Multiprobiotic and control, $(\mathrm{M} \pm \mathrm{m})$.

\begin{tabular}{|c|c|c|c|c|c|}
\hline \multirow{2}{*}{ No } & \multirow{2}{*}{ Value } & Before treatment & After treatment & Control group & \multirow{2}{*}{ Statistic value } \\
\hline & & $(n=36)$ & $(n=36)$ & $(n=20)$ & \\
\hline \multirow{3}{*}{1} & \multirow{3}{*}{$\begin{array}{l}\text { Ornithinedecarboxylase activity, } \\
\text { nmol/ml*min }\end{array}$} & \multirow{3}{*}{$23.64 \pm 1.15$} & \multirow{3}{*}{$30.9 \pm 1.11$} & \multirow{3}{*}{$34.47 \pm 0.53$} & $\mathrm{P}_{1-2}<0.05$ \\
\hline & & & & & $\mathrm{P}_{1-3}<0.05$ \\
\hline & & & & & $\mathrm{P}_{2-3}<0.05$ \\
\hline \multirow{3}{*}{2} & \multirow{3}{*}{$\begin{array}{l}\text { Catalase activity, } \\
\mu c a t / l\end{array}$} & \multirow{3}{*}{$0.13 \pm 0.0067$} & \multirow{3}{*}{$0.29 \pm 0.0099$} & \multirow{3}{*}{$0.32 \pm 0.0044$} & $\mathrm{P}_{1-2}<0.05$ \\
\hline & & & & & $\mathrm{P}_{1-3}<0.05$ \\
\hline & & & & & $\mathrm{P}_{2-3}<0.05$ \\
\hline \multirow{3}{*}{3} & \multirow{3}{*}{ Total proteolytic activity , $\mu \mathrm{g} / \mathrm{ml} \mathrm{l}^{*} \mathrm{~min}$} & \multirow{3}{*}{$18.03 \pm 0.13$} & \multirow{3}{*}{$13.7 \pm 0.49$} & \multirow{3}{*}{$11.4 \pm 0.44$} & $\mathrm{P}_{1-2}<0.05$ \\
\hline & & & & & $\mathrm{P}_{1-3}<0.05$ \\
\hline & & & & & $\mathrm{P}_{2-3}<0.05$ \\
\hline \multirow{3}{*}{4} & \multirow{3}{*}{ Total antitryptic activity, g/l } & \multirow{3}{*}{$2.44 \pm 0.16$} & \multirow{3}{*}{$4.42 \pm 0.08$} & \multirow{3}{*}{$5.03 \pm 0.03$} & $\mathrm{P}_{1-2}<0.05$ \\
\hline & & & & & $\mathrm{P}_{1-3}<0.05$ \\
\hline & & & & & $\mathrm{P}_{2-3}<0.05$ \\
\hline \multirow{3}{*}{5} & \multirow{3}{*}{ NOS activity, $\mu \mathrm{mol} / \mathrm{l}^{*} \mathrm{~min}$} & \multirow{3}{*}{$3.33 \pm 0.31$} & \multirow{3}{*}{$6.13 \pm 0.16$} & \multirow{3}{*}{$6.41 \pm 0.45$} & $\mathrm{P}_{1-2}<0.05$ \\
\hline & & & & & $\mathrm{P}_{1-3}<0.05$ \\
\hline & & & & & $\mathrm{P}_{2-3}<0.05$ \\
\hline \multirow{3}{*}{6} & \multirow{3}{*}{ Amount of $\mathrm{NO}_{2} ;, \mu \mathrm{mol} / \mathrm{l}$} & & & & $\mathrm{P}_{1-2}<0.05$ \\
\hline & & $6.72 \pm 0.29$ & $7.81 \pm 0.35$ & $8.11 \pm 0.15$ & $\mathrm{P}_{1-3}<0.05$ \\
\hline & & & & & $\mathrm{P}_{2-3}<0.05$ \\
\hline & & & & & $\mathrm{P}_{1-2}<0.05$ \\
\hline 7 & Volume of oxygen modified proteins, a.u. & $0.12 \pm 0.0047$ & $0.06 \pm 0.0021$ & $0.05 \pm 0.0028$ & $\mathrm{P}_{1-3}<0.05$ \\
\hline & & & & & $\mathrm{P}_{2-3}<0.05$ \\
\hline & & & & & $\mathrm{P}_{1-2}<0.05$ \\
\hline 8 & Volume of TBA-reactants, $\mu \mathrm{mol} / \mathrm{l}$ & $64.64 \pm 7.79$ & $40.47 \pm 1.47$ & $36.54 \pm 0.75$ & $\mathrm{P}_{1-3}<0.05$ \\
\hline & & & & & $\mathrm{P}_{2-3}<0.05$ \\
\hline & & & & & $\mathrm{P}_{1-2}<0.05$ \\
\hline 9 & Superoxide dismutase activity, u/g & $0.12 \pm 0.0039$ & $0.18 \pm 0.0019$ & $0.19 \pm 0.0019$ & $\mathrm{P}_{1-3}<0.05$ \\
\hline & & & & & $\mathrm{P}_{2-3}<0.05$ \\
\hline & & & & & $\mathrm{P}_{1-2}<0.05$ \\
\hline 10 & Intermediate mass molecules, a.u. & $0.2 \pm 0.0064$ & $0.11 \pm 0.0054$ & $0.1 \pm 0.0042$ & $\mathrm{P}_{1-3}<0.05$ \\
\hline & & & & & $\mathrm{P}_{2-3}<0.05$ \\
\hline
\end{tabular}

evaluation of body antioxidant system. It has been established that activity of catalase and superoxide dismutase in oral fluid is significantly decreased in patients with generalized chronic periodontitis. A significant increase of catalase and superoxide dismutase in oral fluid of all patients with generalized chronic periodontitis has been noted after treatment with "Simbiter omega" multiprobiotic, as compared to the values before treatment (Figure 3).

\section{References}

1. Aspriello SD, Zizzi A, Lucarini G, Rubini C, Faloia E, Boscaro M, et al. Vascular endothelial growth factor and microvessel density in periodontitis patients with and without diabetes. J Periodontol. 2009; 80(11): 1783-9.

2. Grudyanov AI, Dmitriyeva NA, Fomenko EV. Administration of probiotics in complex treatment of inflammatory periodontal diseases. Moscow: MIA LLC; 2006.

3. Lorenzi T, Niţulescu EA, Zizzi A, Lorenzi M, Paolinelli F, Aspriello SD, et al. The novel role of HtrA1 in gingivitis, chronic and aggressive periodontitis. PLoS One. 2014; 9(6): e96978.
4. Armitage GC. Development of a classification system for periodontal diseases and conditions. Ann Periodontol. 1999; 4(1): 1-6.

5. Yankovskiy DS, Shyrobokov VP, Volosovets AP, et al. (2013) Human microbiome and current methods of its improvement: the literature review. Journal of NAMS of Ukraine. 19(4): 411-420.

6. Danylevskiy MF, Borysenko AV. Periodontal diseases. Kyiv: Health; 2000.

7. Dubinina EE, Burmistrov SO. Oxidative modification of proteins of human serum. Method of determining. Questions of medicinal chemistry. 1995; 1: 24-26.

8. Stalnaya ID, Garishvili TG. Method of measuring of malonic dialdehyde by thiobarbituric acid. Contemporary methods in biochemistry. Moscow: Medicine; 1977.

9. Korolyuk MA, Ivanova LI, Mayorova IG. Method for determining catalase activity. Laboratory issue. 1988; 1: 19-26.

10. Brusov OS, Gerasimov AM, Panchenko LF. Impact of natural inhibitors of radical reactions onto adrenaline auto-oxidation. Newsletter of experimental biology and medicine. 1976; 1: 33-35.

11. Gabrielyan NI, Lipatova VI. Actual experience with index of 
intermediate mass molecules in blood for diagnostics of nephrologica disease in children. Lab issue. 1984; 3: 138-140.

12. Kamyshnikov VS. Handbook on clinicobiochemistry laboratory diagnostics. Minsk; 2000.

13. Berkalo LV, Bobovych OV, Bobrova NO. Methods of clinical and experimental studies in medicine. Poltava: Polimet; 2003.

14. Khramov VA. Simple method of determining ornithinedecarboxylase activity in human mixed saliva. Clinical laboratory diagnostics. 1997; 4: 14-15.

15. Dyachenko VF, Biryukova SV, Starobinets ZG, at al. Laboratory diagnostics of pyoinflammatory diseases, caused by asporogenic microorganisms. Methodological guidelines. Kharkiv; 2000.

16. Aspriello SD, Zizzi A, Tirabassi G, Buldreghini E, Biscotti T, Faloia E et al. Diabetes mellitus-associated periodontitis: differences between type 1 and type 2 diabetes mellitus. J Periodontal Res. 2011; 46(2): 164-9.

17. Lucarini G, Zizzi A, Aspriello SD, Ferrante L, Tosco E, Lo Muzio L, et al. Involvement of vascular endothelial growth factor, CD44 and CD133 in periodontal disease and diabetes: an immunohistochemical study.J Clin Periodontol. 2009; 36(1): 3-10.

18. Myerson FZ, Pshennikova MG. Adaptation to stress situations and physical activities. Moscow: Medicine; 1988.

19. Myerson FZ. Pathogenesis and prevention of stress and ischemic heart injuries. Moscow: Medicine; 1984.

20. Bilenko MV. Ischemic and reperfusion injuries of organs (molecular mechanisms, modes of injury and treatment). Moscow: Medicine; 1989. 